\title{
External validation of a COPD diagnostic questionnaire
}

\author{
D. Kotz ${ }^{*, \#}$, P. Nelemans ${ }^{\#, \uparrow}$, C.P. van Schayck, ${ }^{\star, \#}$ and G.J. Wesseling ${ }^{\#,+}$
}

ABSTRACT: The aim of the present study was to determine the external validity of a recently developed questionnaire for the identification of patients at increased risk of airflow limitation in smokers from the general population in the provinces of Dutch and Belgian Limburg (regions surrounding Maastricht, the Netherlands).

As part of a study on the early detection of airflow limitation and subsequent smoking cessation treatment (International Standard Randomised Controlled Trial Number: 64481813), the recently developed chronic obstructive pulmonary disease (COPD) diagnostic questionnaire was used in current smokers aged $40-70$ yrs, with a smoking history of $\geqslant 10$ pack-yrs, who reported one or more respiratory symptom (cough, sputum production or dyspnoea), but who had no diagnosis of a respiratory disease (COPD or asthma). Spirometry performed according to American Thoracic Society/European Respiratory Society criteria served as a reference test.

Of the 676 subjects who entered the analyses, 398 showed normal lung function and 278 had a diagnosis of COPD (post-bronchodilator forced expiratory volume in one second/forced vital capacity of $<\mathbf{0 . 7 0}$ ). The ability of the COPD diagnostic questionnaire to discriminate between subjects with and without COPD was poor (area under the receiver operating characteristic curve of 0.65).

In a high-risk population consisting of middle-aged current smokers with a smoking history of $\geqslant 10$ pack-yrs, the chronic obstructive pulmonary disease diagnostic questionnaire is probably not useful as a diagnostic tool for the identification of patients with an increased risk of airflow limitation.

KEYWORDS: Chronic obstructive pulmonary disease, diagnostic questionnaire, early diagnosis, external validation, spirometry

hronic obstructive pulmonary disease (COPD) is associated with a high personal and societal burden and mortality. Projections for 2020 indicate further increase in global COPD mortality, making COPD the third leading cause of death [1]. Underdiagnosis of COPD is a worldwide problem [2]. Most patients present to their doctor for various other reasons but often have respiratory symptoms, and, in those who present with respiratory symptoms, COPD is not always diagnosed [3]. Owing to the irreversible and progressive nature of the disease, early identification of COPD and subsequent treatment is important. However, widespread spirometric testing for early detection without pre-selection of at-risk patients may result in wasting healthcare resource utilisation $[4,5]$.

The efficiency of early detection of COPD can be enhanced (in terms of detection rate and accuracy) by using simple self-administered questionnaires to identify persons in whom airflow limitation is likely [6, 7]. PRICE and co-workers
$[8,9]$ have developed a new symptom-based questionnaire for the identification of patients at increased risk of airflow limitation. Their COPD diagnostic questionnaire discriminates between subjects with and without airflow limitation. These authors concluded that their questionnaire could be used to identify patients with a high likelihood of showing airflow limitation, and that combining it with spirometry could help to improve the efficiency and accuracy of COPD diagnosis in primary care $[8,9]$. The questionnaire has recently been implemented in the guideline for chronic airways diseases of the International Primary Care Airways Group [10]. The guideline recommends use of the questionnaire in current and former smokers aged $\geqslant 40$ yrs who present with respiratory symptoms but no prior history of respiratory disease or current regular respiratory treatment.

Before a new diagnostic questionnaire can be accepted and applied reliably in clinical practice, the analysis of the underlying model must be
AFFILIATIONS

*Depts of General Practice,

Epidemiology,

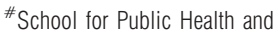
Primary Care (CAPHRI), Maastricht

University, and

+Dept of Respiratory Medicine,

Maastricht University Hospital,

Maastricht, The Netherlands.

CORRESPONDENCE

D. Kotz

Dept of General Practice

School for Public Health and Primary Care (CAPHRI)

Maastricht University

P.0. Box 616

6200 MD Maastricht

The Netherlands

Fax: 31433619344

E-mail: d.kotz@hag.unimaas.nl

Received:

June 202007

Accepted after revision:

September 262007

SUPPORT STATEMENT

This study was supported by grants from the Dutch Asthma Foundation

(Leusden, the Netherlands), Partners

in Care Solutions for COPD (the

Netherlands), and Maastricht

University Hospital (Maastricht, the Netherlands)

STATEMENT OF INTEREST

A statement of interest for C.P. van Schayck can be found at www.erj.ersjournals.com/misc/ statements.shtml

European Respiratory Journal Print ISSN 0903-1936

Online ISSN 1399-3003 
repeated on new data collected from an appropriate sample of subjects in a different setting [11-13]. This process is called external validation. The aim of the present study was to determine the validity of the COPD diagnostic questionnaire in current smokers from the general population in the provinces of Dutch and Belgian Limburg.

\section{METHODS}

\section{Development of the COPD diagnostic questionnaire in the original sample}

The development of the COPD diagnostic questionnaire has been described in detail elsewhere [8, 9]. In brief, 818 participants completed a list of questions and underwent spirometry. COPD was defined according to the Global Initiative for Chronic Obstructive Lung Disease (GOLD) guideline [14] by a post-bronchodilator (post-BD) forced expiratory volume in one second (FEV1)/forced vital capacity (FVC) of $<0.70$. Multiple logistic regression models were constructed in order to identify eight items for discriminating between persons with and without COPD. The scoring system of the questionnaire permits calculation of an overall COPD risk based on the weighted scores for these items. The sum score ranges 0 38 points. According to the manual of the questionnaire, subjects can be classified as being at high ( $>19.5$ points), moderate (16.5-19.5 points) or low (0-16.5 points) risk of COPD. The area under the receiver operating characteristic (ROC) curve (ROCAUC) was 0.82. The diagnostic odds ratios (DORs) of the two cut-off points, 16.5 and 19.5, were 5.5 and 4.8; calculation based on that of PRICE et al. [9].

\section{External validation sample}

This external validation study was part of a study on the early detection of airflow limitation and subsequent smoking cessation treatment. The trial protocol was approved by the medical ethics committee of Maastricht University/Maastricht University Hospital (Maastricht, the Netherlands) and registered (International Standard Randomised Controlled Trial Number: 64481813) at the Netherlands Trial Register (Dutch Cochrane Centre, Academic Medical Centre, Amsterdam, the Netherlands).

Subjects were recruited from the general population (through advertisements in a local newspaper, flyers, posters and mailings to households) and from primary care practices (during consultations and through posters and personalised mailings) in the provinces of Dutch and Belgian Limburg (the region surrounding Maastricht, the Netherlands) during the period January 2005 to December 2006. Smokers aged 3570 yrs who were current smokers but motivated to quit smoking were invited to take part in a study on individual counselling and medication for smoking cessation. The external validation of the COPD diagnostic questionnaire was performed in current smokers aged 40-70 yrs.

Eligibility was screened during an initial telephone interview. Inclusion criteria were: a smoking history of $\geqslant 10$ pack-yrs; being motivated to stop smoking; being competent to read and speak Dutch; and reporting a respiratory symptom, defined as an affirmative answer to at least one of the following three questions. 1) "Do you cough regularly?" 2) "Do you cough up phlegm (sputum) when you don't have a cold?" 3) "Have you been shorter of breath lately?" Exclusion criteria were: evidence of a prior respiratory diagnosis, defined by an affirmative answer to the following question: "Do you have COPD, chronic bronchitis, asthma or asthmatic bronchitis?" They were also not permitted to have undergone a lung function test (spirometry) during the preceding 12 months. Since candidates were being screened for taking part in a trial on smoking cessation, one or more contraindications to using the smoking cessation medication (nortriptyline) were also criteria for exclusion, among others the current use of antidepressants. Nortriptyline is a tricyclic antidepressant which should not be used for smoking cessation in conjunction with another antidepressant.

After the initial telephone interview, participant information, along with the informed consent form and the COPD diagnostic questionnaire, was sent to eligible subjects and a date was fixed for spirometry. Linguistic validation of the original English COPD diagnostic questionnaire for use in Dutch-speaking people had been performed by an experienced international research institute (Mapi Research Institute, Lyon, France). This process comprised conceptual definition of items, forward and backward translation, and testing. Subjects filled in the questionnaire at home and handed it in during the spirometry visit, during which they also signed the informed consent form.

Spirometry was performed by two qualified research assistants under the permanent supervision of a pulmonologist (G.J. Wesseling) according to the criteria of the American Thoracic Society/European Respiratory Society task force on standardisation of lung function testing $[15,16]$ using a Vitalograph ${ }_{\mathbb{R}}$ 2120 (Vitalograph, Buckingham, UK). After a minimum of three acceptable and reproducible FVC manoeuvres, a bronchodilator (500 $\mu$ g terbutaline) was administered to the subject in preparation for the reversibility test. After $15 \mathrm{~min}$, another series of three FVC manoeuvres was performed. All spirometric test results were independently validated by a pulmonologist (G.J. Wesseling) and a specialised lung function laboratory assistant who was not involved in the trial. Both were blinded to the scores on the COPD questionnaire. In the case of initial disagreement, consensus was obtained during reexamination. As in the study of PRICE and co-workers $[8,9]$, a diagnosis of COPD was confirmed by a post-BD FEV1/FVC of $<0.70$, according to the international GOLD guideline [14].

\section{Statistical analyses}

The sum score for the COPD questionnaire was calculated based on the original scoring system [9]. The ROCAUC, sensitivity, specificity and DOR were then calculated for the two cut-off points presented in the questionnaire manual. A multiple logistic regression model was fitted, including the eight items from the COPD diagnostic questionnaire as independent variables and COPD as dependent variable in order to compare odds ratios, logistic regression coefficients and corresponding $p$-values between the original sample and the external validation sample.

\section{RESULTS}

\section{Study population}

A summary of the study enrolment protocol is shown in figure 1 . A total of 1,711 subjects were screened for eligibility by telephone. The majority, 1,209 (70\%) subjects, had 
responded to an advertisement, $166(10 \%)$ had been motivated by their general practitioner to take part in the study and 196 $(11 \%)$ by a related study participant, and 140 (8\%) had responded to flyers and posters or their motivation was not recorded. Of these, 1,052 subjects were found to be eligible and were invited for spirometry. Spirometry was performed and the COPD diagnostic questionnaire was collected from 826 subjects; the remaining 226 subjects cancelled their spirometry appointment beforehand or did not show up. Of the spirometric test results, $13 \%$ (110 out of 826 ) were found to be invalid and so had to be excluded from the analyses. This proportion of invalid test results was higher than in the original study ( 80 out of $898 ; 9 \%$ ). The 110 subjects with invalid test results did not differ from those with valid test results with regard to sex, age, smoking history and COPD diagnostic questionnaire sum score (results of statistical tests not reported). In total, 40 subjects were excluded due to missing data for one or more of the items of the COPD diagnostic questionnaire. The characteristics of these subjects did not differ from those with complete data for the COPD diagnostic questionnaire except for the post-BD FVC, which was significantly lower (mean \pm SD $3.51 \pm 0.76$ versus $3.98 \pm 0.95 \mathrm{~L} ; \mathrm{p}=0.002$ ). Complete valid data from 676 subjects were used in the analyses.

\section{Characteristics of the external validation sample}

Of the 676 study subjects, 378 (59\%) exhibited normal lung function (mean post-BD FEV1/FVC of 77.9 ; mean post-BD

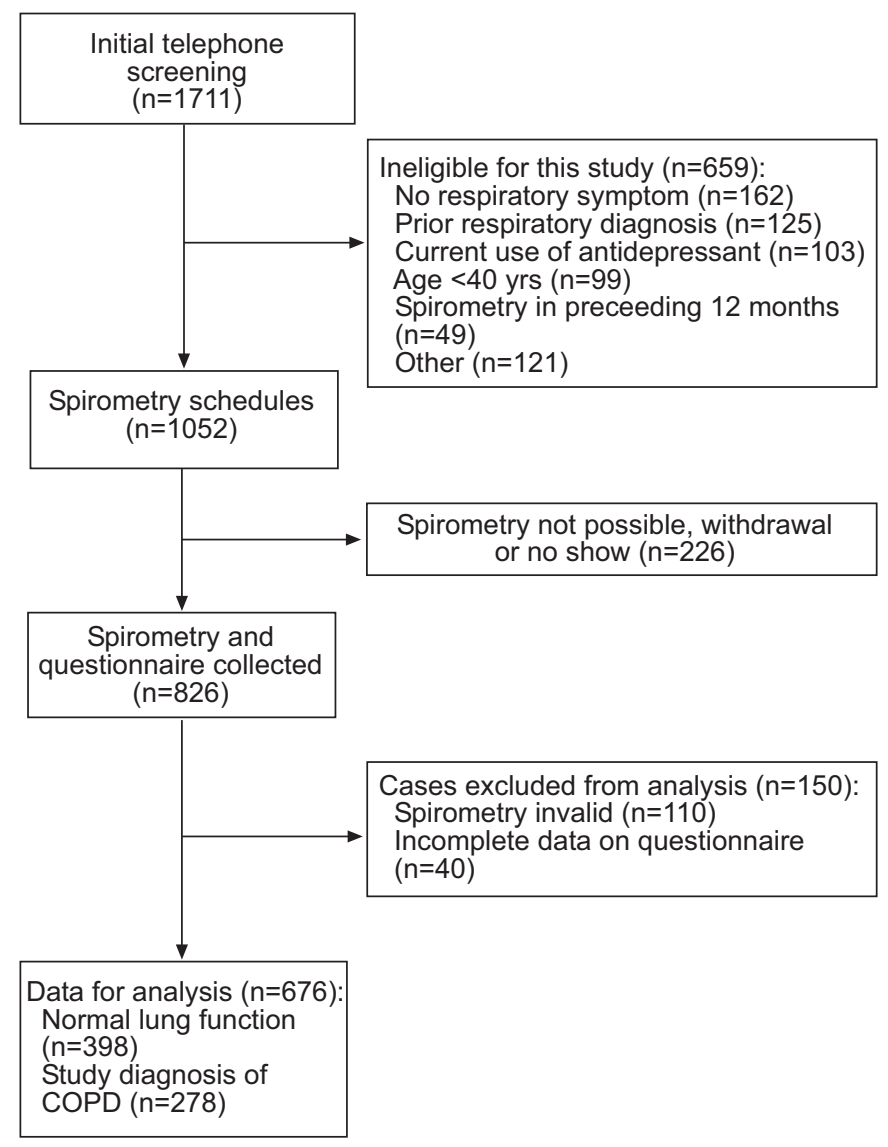

FIGURE 1. A flow diagram to show study enrolment. COPD: chronic obstructive pulmonary disease.
FEV1 of $94.1 \%$ of the predicted value) and $278(41 \%)$ had a diagnosis of COPD (mean post-BD FEV1/FVC of 61.9; mean post-BD FEV1 of $79.7 \%$ pred). In the latter group, airflow limitation according to GOLD criteria [14] was mild in 142 (21\%) subjects, moderate in $119(18 \%)$ and severe-to-very severe in $17(3 \%)$. All other characteristics are shown in table 1. On average, subjects from the present external validation sample were younger and exhibited slightly worse lung function parameters than those from the original sample. The prevalence of COPD was much higher (41 versus 19\%). All subjects were current smokers, whereas only $45 \%$ of the original sample had been current smokers. This difference in smoking status probably explains the large difference in mean smoking history (40 versus 26 pack-yrs in the original sample). The external validation sample was split into two subsets according to the study diagnosis in order to compare differences in characteristics. This is also shown in table 1 (without calculation of statistical differences). Smokers with COPD were more likely to be older and male and to show greater cumulative cigarette consumption.

\section{Discriminative ability of the COPD diagnostic questionnaire}

When applying the two cut-off points from the questionnaire manual (16.5 points for a low risk and 19.5 points for a high risk), 127 (19\%) out of 676 subjects were categorised as having a low risk of COPD, $183(27 \%)$ as having a moderate risk and $366(54 \%)$ as having a high risk. Within these three risk categories, the observed prevalence of COPD was $24 \%$ (30 out of 127 subjects) in the low-risk category, 36\% (65 out of 183) in the moderate-risk category and 50\% (183 out of 366) in the high-risk category. The ability of the COPD diagnostic questionnaire to discriminate between subjects with and

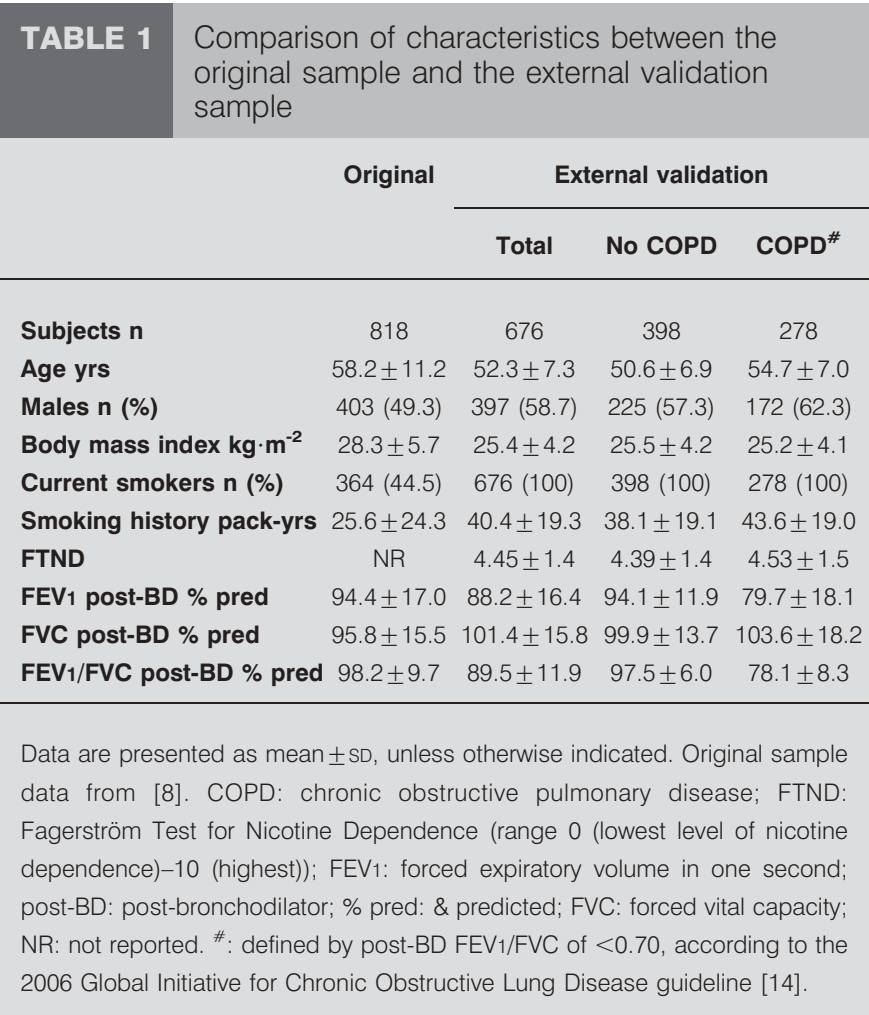




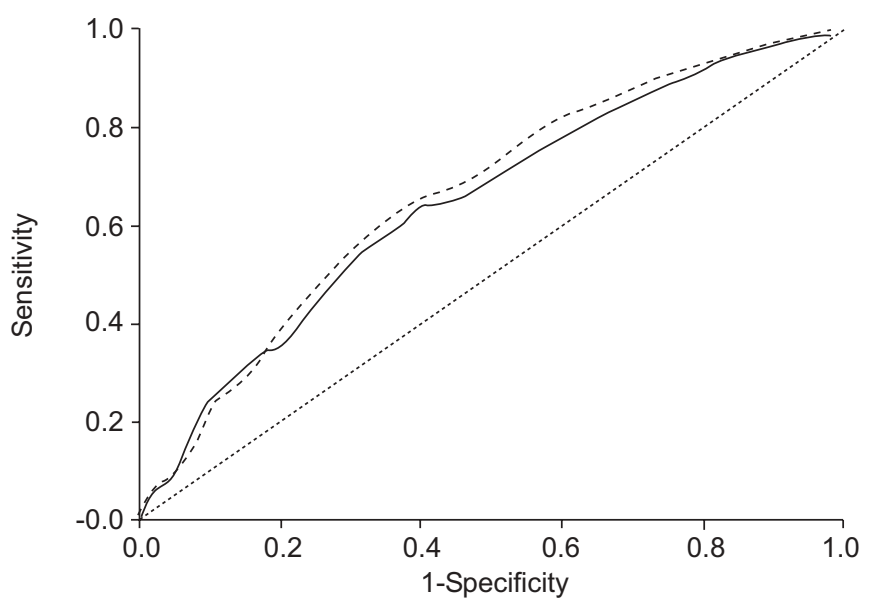

FIGURE 2. Receiver operating characteristic curves for predictors of chronic obstructive pulmonary disease (COPD) in the external validation sample. sum score of the COPD diagnostic questionnaire (area under the curve (AUC) 0.65); ------: age alone (AUC 0.67); …..: reference line.

without COPD is shown graphically by the ROC curve in figure 2. The solid line represents levels of sensitivity and false positive rate for all cut-off points for the sum score of the questionnaire. The ROCAUC was considerably lower (0.65) than in the original sample (0.82). Sensitivity, specificity and DOR for the cut-off point 16.5 were $89.2 \%, 24.4 \%$ and 2.67 , respectively. For the cut-off point 19.5 , these parameters were $65.8 \%, 54.0 \%$ and 2.26 , respectively.

\section{Refit of the multiple logistic regression model}

The results of the multiple logistic regression analysis that modelled the probability of having COPD versus not having COPD are shown in table 2. Significant associations were observed for age, body mass index and the two items "phlegm in the morning" and "any wheeze". The other items were associated with odds ratios that did not differ significantly from 1.

Since age seemed to be the most important predictor of the disease, another ROC curve was calculated, with age as the single factor for predicting COPD. This curve is shown in figure 2 . The ROCAUC was 0.67 , meaning that the variable age alone showed similar discriminative ability to the sum score from the COPD diagnostic questionnaire in the present sample.

\section{DISCUSSION}

In the present study, the validity of a recently developed COPD diagnostic questionnaire was tested in 676 current smokers from the general population in Dutch and Belgian Limburg. The discriminative ability of the questionnaire was poor (ROCAUC of 0.65).

The COPD diagnostic questionnaire was developed in order to improve the efficiency and accuracy of COPD diagnosis in primary care by discriminating between subjects with and without airflow limitation [8,9]. When applying the cut-off points from the manual, the questionnaire reached sensitivities of 65.8 and $89.2 \%$ (at cut-off points of 19.5 and 16.5, respectively). However, the corresponding specificities were

\begin{tabular}{|c|c|c|c|c|c|c|}
\hline \multirow[t]{3}{*}{ TABLE 2} & \multicolumn{6}{|c|}{$\begin{array}{l}\text { Comparison of multiple regression models } \\
\text { between the original sample }{ }^{\#} \text { and the external } \\
\text { validation sample }\end{array}$} \\
\hline & & \multicolumn{2}{|c|}{ Original } & \multicolumn{3}{|c|}{ External validation } \\
\hline & & $\mathrm{OR}^{+}$ & $\mathrm{p}$-value & Subjects & n OR $(95 \% \mathrm{Cl})^{+}$ & $\mathrm{p}$-value \\
\hline \multicolumn{7}{|l|}{ Age group yrs } \\
\hline $40-49^{5}$ & & 1.00 & & 276 & 1.00 & \\
\hline $50-59$ & & 2.20 & 0.022 & 265 & $1.99(1.38-2.88)$ & $<0.001$ \\
\hline $60-69$ & & 4.73 & $<0.001$ & 133 & $2.92(1.85-4.61)$ & $<0.001$ \\
\hline$\geqslant 70$ & & 7.77 & $<0.001$ & 2 & NA & NA \\
\hline \multicolumn{7}{|l|}{ BMI $\mathbf{k g} \cdot \mathrm{m}^{-2}$} \\
\hline$<25.4^{\S}$ & & 1.00 & & 386 & 1.00 & \\
\hline $25.4-29.7$ & & 0.44 & 0.002 & 191 & $0.66(0.45-0.97)$ & 0.033 \\
\hline$>29.7$ & & 0.35 & $<0.001$ & 99 & $0.57(0.35-0.94)$ & 0.026 \\
\hline \multicolumn{7}{|c|}{$\begin{array}{l}\text { Smoking history pack- } \\
\text { yrs }\end{array}$} \\
\hline$<15^{\S}$ & & 1.00 & & 26 & 1.00 & \\
\hline $15-24$ & & 1.63 & 0.112 & 106 & $0.74(0.29-1.92)$ & 0.537 \\
\hline $25-49$ & & 1.99 & 0.010 & 367 & $1.11(0.46-2.63)$ & 0.822 \\
\hline$\geqslant 50$ & & 4.05 & $<0.001$ & 177 & $1.66(0.67-4.10)$ & 0.271 \\
\hline Weather affec & ts cough ${ }^{f}$ & 1.68 & 0.089 & 138 & $0.87(0.58-1.31)$ & 0.502 \\
\hline Phlegm witho & ut a cold ${ }^{f}$ & 1.81 & 0.013 & 486 & $1.47(0.7-2.22)$ & 0.068 \\
\hline Phlegm in the & morning ${ }^{f}$ & 0.54 & 0.022 & 253 & $0.67(0.46-0.99)$ & 0.045 \\
\hline Wheeze ${ }^{\# \#}$ & & 2.08 & 0.001 & 535 & $1.74(1.14-2.68)$ & 0.011 \\
\hline $\begin{array}{l}\text { Present/previ } \\
\text { gies }^{f}\end{array}$ & us aller- & 0.52 & 0.005 & 154 & $0.98(0.67-1.47)$ & 0.994 \\
\hline
\end{tabular}

Original sample data from [9]. OR: odds ratio; $\mathrm{Cl}$ : confidence interval; $\mathrm{BMI}$ body mass index; NA: not applicable (insufficient number of cases in category). \#: $n=798 ; \because: n=676 ;{ }^{+}$: for having chronic obstructive pulmonary disease (COPD; i.e. forced expiratory volume in one second (FEV 1 )/forced vital capacity (FVC) of $<0.70$ with respiratory symptoms) versus not having COPD (i.e. FEV1/ FVC of $\geqslant 0.70)$; ${ }^{s}$ : reference; ${ }^{f}$ : yes versus no; ${ }^{\# \#}$ : any versus never.

only 54.0 and $24.4 \%$, respectively. This means that, at the cutoff point of 16.5 , almost nine out of 10 subjects with airflow limitation were correctly identified by the questionnaire (true positive). However, at the same time, almost three-quarters of subjects without airflow limitation were incorrectly classified by the questionnaire as having COPD (false positive). A diagnostic test that is intended to discriminate between subjects with and without a disease should combine high levels of sensitivity and specificity. The ROCAUC, however, was very low at 0.65 (a ROCAUC of 0.50 indicates a totally uninformative test [11]). The combination of the eight items of the COPD diagnostic questionnaire did not perform better than the item age alone (ROCAUC of 0.67). Another indicator for the discriminative ability of the questionnaire, the DOR, was also very low at 2.67 at the cut-off point of 16.5. The DOR ranges $0-\infty$, with a value of 1 meaning that a test does not discriminate between subjects with the disease under study and those without it [17].

Why does the COPD diagnostic questionnaire show such a low external validity? It is not unusual that newly developed diagnostic questionnaires perform more poorly when evaluated 
in an external sample of subjects in a different setting. An important reason for the poor performance of the COPD diagnostic questionnaire is that the present sample differs from the original one. This external validation formed part of a study on the early detection of smokers with airflow limitation and subsequent smoking cessation treatment. Subjects from the present sample were all current smokers with a smoking history of $\geqslant 10$ pack-yrs (mean 40 pack-yrs). Only current smokers were included as smoking is by far the most important risk factor for COPD, and smoking cessation is the single most effective way of affecting outcome in patients who have been positively screened [18, 19]. In the sample of PRICE and co-workers [8, 9], only $45 \%$ were current smokers and the mean cumulative cigarette consumption was much lower (26 pack-yrs).

An explanation for the difference in performance may be the difference in smoking status between the present external validation sample and the original sample. The present authors suggest that the COPD diagnostic questionnaire discriminates between current smokers and former or nonsmokers, rather than between subjects with and without airflow limitation. Previous research has shown that smoking exerts acute effects on respiratory symptoms. For example, in the Nord-Trøndelag Health Study, which is a population-based study of $>65,000$ subjects, a significantly higher proportion of smokers compared to ex-smokers reported symptoms of wheezing, breathlessness, daily coughing and coughing with phlegm [20]. Respiratory symptoms were also very common among smokers from the present study; $91 \%$ of the subjects initially screened reported one of the three symptoms cough, sputum production and shortness of breath, and only $9 \%$ had none of these three symptoms. It is unlikely that exclusion of this small proportion of subjects from the present study led to a selective study sample. Since smoking correlates so closely with the presence of respiratory symptoms, the present authors believe that the development and use of diagnostic questionnaires for COPD should be undertaken separately in current and former smokers. Ultimately, such a questionnaire is not intended to discriminate between the presence and absence of respiratory symptoms, but rather between having COPD and not having COPD. It would be interesting to re-analyse the performance of the questionnaire in the two subgroups of smokers and former smokers from the original dataset.

Apart from their smoking status, subjects from the present external validation sample were younger and showed slightly worse lung function parameters than those from the original sample. Furthermore, the proportion of invalid test results was higher (17 versus 9\%). This may be due to a greater measurement error, or to more conservative criteria for the assessment of quality. Nevertheless, it is unlikely that the exclusion of subjects with invalid test results affected the present results since subjects with invalid test results showed similar characteristics to those with valid test results. The prevalence of COPD was much higher in the present sample $(41 \%)$ than in the original one $(19 \%)$. However, it is unlikely that this higher prevalence affected the discriminative ability of the questionnaire since ROC curves and the DOR are not dependent upon the prevalence of disease (although they are influenced by the spectrum of disease severity) [17, 21].
Based on the results of the present external validation study, it is concluded that the chronic obstructive pulmonary disease diagnostic questionnaire is probably not useful as a diagnostic tool for the identification of patients at increased risk of airflow limitation. The questionnaire does not discriminate between subjects with and without airflow limitation in a high-risk population (i.e. middle-aged current smokers with a smoking history of $\geqslant 10$ pack-yrs). The present study highlights the importance of external validation of a newly developed diagnostic instrument prior to its implementation in guidelines and daily practice.

\section{ACKNOWLEDGEMENTS}

The authors gratefully acknowledge the contributions of $\mathrm{K}$. van der Meer (telephonic screening and logistics; Maastricht University, Maastricht, the Netherlands), A. van de Voorde (Maastricht University Hospital, Maastricht, the Netherlands) and E. de Goeij (spirometry; Maastricht University, Maastricht, the Netherlands), M. Aussems (validation of spirometric test results), W. de Goeij (database construction; Infoficiency, Ulestraten, the Netherlands) and P. Rinkens (data cleaning; Maastricht University, Maastricht, the Netherlands).

\section{REFERENCES}

1 Murray CJ, Lopez AD. Alternative projections of mortality and disability by cause 1990-2020: Global Burden of Disease Study. Lancet 1997; 349: 1498-1504.

2 Pauwels PRA, Rabe KF. Burden and clinical features of chronic obstructive pulmonary disease (COPD). Lancet 2004; 364: 613-620.

3 van Schayck CP, van der Heijden FMMA, van den Boom G, Tirimanna PRS, van Herwaarden CLA. Underdiagnosis of asthma: is the doctor or the patient to blame? The DIMCA project. Thorax 2000; 55: 562-565.

4 Wilt TJ, Niewoehner D, Kim C-B, et al., Use of Spirometry for Case Finding, Diagnosis, and Management of Chronic Obstructive Pulmonary Disease (COPD). Evidence Report/Technology Assessment No. 121. Agency for Healthcare Research and Quality. Publication No. 05E017-2. Rockville, Agency for Healthcare Research and Quality, 2005.

5 Enright P. Does screening for COPD by primary care physicians have the potential to cause more harm than good? Chest 2006; 129: 833-835.

6 van Schayck CP, Loozen JM, Wagena E, Akkermans RP, Wesseling GJ. Detecting patients at a high risk of developing chronic obstructive pulmonary disease in general practice: cross sectional case finding study. BMJ 2002; 324: 1370-1374.

7 van Schayck CP, Halbert RJ, Nordyke RJ, Isonaka S, Maroni J, Nonikov D. Comparison of existing symptombased questionnaires for identifying COPD in the general practice setting. Respirology 2005; 10: 323-333.

8 Price DB, Tinkelman DG, Halbert RJ, et al. Symptom-based questionnaire for identifying chronic obstructive pulmonary disease in smokers. Respiration 2006; 73: 285-295.

9 Price DB, Tinkelman DG, Nordyke RJ, Isonaka S, Halbert RJ, for the COPD Questionnaire Study Group, Scoring system and clinical application of COPD diagnostic questionnaires. Chest 2006; 129: 1531-1539. 
10 Grouse L, DeWeerdt S, eds. IPAG Diagnosis and Management Handbook. Chronic Airways Diseases. A Guide for Primary Care Physicians. Edgewater, MCR Vision, Inc., 2005.

11 Knottnerus JA. The Evidence Base of Clinical Diagnosis. London, BMJ Books, 2002.

12 Altman DG, Royston P. What do we mean by validating a prognostic model? Stat Med 2000; 19: 453-473.

13 Justice AC, Covinsky KE, Berlin JA. Assessing the generalizability of prognostic information. Ann Intern Med 1999; 130: 515-524.

14 Global Initiative for Chronic Obstructive Lung Disease, Global Strategy for the Diagnosis, Management, and Prevention of Chronic Pulmonary Disease. Executive Summary. Bethesda, National Heart, Lung, and Blood Institute/World Health Organization, 2006.

15 Miller MR, Hankinson J, Brusasco V, et al. Standardisation of spirometry. Eur Respir J 2005; 26: 319-338.

16 Miller MR, Crapo R, Hankinson J, et al. General considerations for lung function testing. Eur Respir J 2005; 26: 153-161.
17 Glas AS, Lijmer JG, Prins MH, Bonsel GJ, Bossuyt PMM. The diagnostic odds ratio: a single indicator of test performance. J Clin Epidemiol 2003; 56: 1129-1135.

18 Anthonisen NR, Connett JE, Kiley JP, et al. Effects of smoking intervention and the use of an inhaled anticholinergic bronchodilator on the rate of decline of FEV1. The Lung Health Study. J Am Med Assoc 1994; 272: 14971505.

19 Pauwels RA, Buist AS, Calverley PM, Jenkins CR, Hurd SS. Global strategy for the diagnosis, management, and prevention of chronic obstructive pulmonary disease. NHLBI/WHO Global Initiative for Chronic Obstructive Lung Disease (GOLD) Workshop summary. Am J Respir Crit Care Med 2001; 163: 1256-1276.

20 Langhammer A, Johnsen R, Holmen J, Gulsvik A, Bjermer L. Cigarette smoking gives more respiratory symptoms among women than among men. The NordTrondelag Health Study (HUNT). J Epidemiol Community Health 2000; 54: 917-922.

21 Altman DG. Practical Statistics for Medical Research. London, Chapman \& Hall, 1991. 